significant, strictly inducer-dependent antitumor activity in a well-established mouse model of B cell lymphoma.

Conclusions The zinc-finger-based transcriptional control system investigated in this study provides small molecule-inducible control over a therapeutically relevant anti-CD20 CAR in primary $\mathrm{T}$ cells in a time- and dose-dependent manner. The tight regulation of CAR expression will pave the way for safer cellular therapies.

Disclosure Information B. Kotter: A. Employment (full or parttime); Significant; Miltenyi Biotec B.V. \& Co. KG. N. Werchau: A. Employment (full or part-time); Significant; Miltenyi Biotec B.V. \& Co. KG. W. Krueger: A. Employment (full or part-time); Significant; Lentigen Technology Inc. A. Roy: A. Employment (full or part-time); Significant; Lentigen Technology Inc. J. Mittelstaet: A. Employment (full or part-time); Significant; Miltenyi Biotec B.V. \& Co. KG. A. Kaiser: A. Employment (full or part-time); Significant; Miltenyi Biotec B. V. \& Co. KG.

\section{P01.23 CREATING A CELL-CULTURE BASED REPORTER SYSTEM FOR THE EVALUATION OF MOLECULAR SIGNALING MECHANISMS OF INHIBITORY CHIMERIC ANTIGEN RECEPTORS}

MA Funk*, A De Sousa Linhares, C Battin, K Radakovics, J Leitner, P Steinberger. Medical University of Vienna, Vienna, Austria

\subsection{6/jitc-2020-ITOC7.35}

Background Adoptive transfer of $\mathrm{T}$ cells expressing chimeric antigen receptors (CARs) is a novel treatment option for patients with B-cell lineage derived cancers. Other cancer entities are less successfully targeted due to the lack of antigens that are expressed on cancer cells but not healthy tissue cells. One way to address this issue is the concept of inhibitory chimeric antigen receptors (iCARs) that deliver an inhibitory signal upon antigen encounter on off-target cells. The activating effect of CARs depends on a multitude of factors including expression levels, affinity, different signaling domains and steric effects. It has to be expected that co-expression of an iCAR would result in even more complexity. Consequently, there is a demand for a robust high-throughput cellular system to evaluate iCAR-formats.

Materials and Methods Our approach is based on a previously published Jurkat based triple parameter reporter cell (TPR) system. This setup allows for molecular monitoring of the $\mathrm{T}$ cell activation state by measuring fluorescent reporter gene expression via flow cytometry. Inhibitory effects of receptors are determined as the ratio of cellular geometric mean fluorescent intensity (gMFI) in the presence of the inhibitory ligand versus without inhibitory ligand.

Results To test if inhibitory receptors would measurably reduce reporter activation, PD-1 as a well-characterized inhibitory receptor was expressed on Jurkat TPRs. When PD-L1 was present during stimulation reporter activation was reduced by 26-34\% proving feasibility of our approach. Intracellular domains of other inhibitory receptors including BTLA, ILT-2 and KIR2DL1 were evaluated. All three domains outperformed PD-1 in a series of experiments with a mean reduction of gMFI by $48-57 \%, 50-53 \%$ and $38-41 \%$ respectively. To assess if the reporter platform could be used to study downstream signaling pathways of inhibitory constructs we created a SHP-2 knock-out reporter cell line using the
CRISPR/Cas9 gene editing technique. To simulate different degrees of activating signal strength, peptide-MHC complex recognizing activating receptors were created. The reporter activation correlated with the concentrations of peptide in the stimulation cultures. Co-expression of iCAR and CAR could be achieved using selection for two separate antibiotic resistance genes introduced into the respective vector. Preliminary experiments showed greatly reduced inhibitory efficacy of iCAR molecules due to an adhesion effect resulting from the high affinity extracellular domain of the iCARs that lead to tighter cell-cell contact and stronger stimulation through the CAR.

Conclusions We present a highly flexible and controllable Jurkat-based reporter cell platform for the thorough study of inhibitory signaling mechanisms. This project was supported by the Austrian Science Fund, FWF; Project P32411

Disclosure Information M.A. Funk: None. A. De Sousa Linhares: None. C. Battin: None. K. Radakovics: None. J. Leitner: None. P. Steinberger: None.

\section{P01.24 THE SELECTIVE HDAC6 INHIBITOR ITF3756 INCREASES THE DIFFERENTIATION TO CENTRAL MEMORY T CELLS WITH REDUCED EXHAUSTION PHENOTYPE}

C Ripamonti, C Steinkuhler, G Fossati*. Italfarmaco SpA, Cinisello Balsamo, Italy

\subsection{6/jitc-2020-ITOC7.36}

Background Central memory $\mathrm{T}$ cells show superior persistence and antitumor immunity compared to effector memory and effector $\mathrm{T}$ cells. $\mathrm{T}$ effector cells respond quickly to tumors, but they are terminally differentiated and undergo apoptosis upon killing activity. $\mathrm{T}$ memory differentiate rapidly into $\mathrm{T}$ effector cells and maintain a pool of cells that can continuously differentiate thus sustaining a more lasting response. In adoptive cell therapy (ACT), T cells infused into patients may have a limited time of activity if they are terminally differentiated, and may rapidly undergo exhaustion and apoptosis. The development of new strategies based on novel agents able to generate memory $\mathrm{T}$ cells ex-vivo is important for a successful clinical application of ACT.We have studied the effect of a potent and selective HDAC6 inhibitor, ITF3756, on CD8 T cells differentiation during an in vitro induced exhaustion process.

Materials and Methods To induce exhaustion purified human CD8 + cells were stimulated twice with anti-CD3/CD28 beads (1:2) during 5 days, with or without ITF3756 $1 \mu \mathrm{M}$ or $2 \mu \mathrm{M}$ added at all times of stimulation. At day 3 and 5 the expression of exhaustion, memory and effector $\mathrm{T}$ cells markers were analyzed by flow cytometry. Cells were also collected at day 5 for genes expression analysis. Expression of exhaustion, $\mathrm{T}$ phenotype, metabolic pathway and inflammatory cytokines were investigated by qPCR. Paired two-tailed t-tests was used to determine statistical significance between control versus treatment group at day 3 and 5 in 10 different donors. P-values $\leq 0.05$ were considered significant.

Results ITF3756 $1 \mu \mathrm{M}$ increased significantly the $\mathrm{T}$ central memory phenotype (CD45RO+CD62L +CCR7+) and decreased significantly the $\mathrm{T}$ effector phenotype (CD45RO +CD62L-CCR7-). The expression of CD62L in $\mathrm{T}$ central memory cells was significantly increased in agreement with the high expression of this marker in naïve and memory $\mathrm{T}$ cells. ITF3756 treatment decreased significantly the expression of 
exhaustion markers PD-1 and LAG-3. No effect was observed on TIM-3 expression. In agreement with the data obtained with protein analysis, treatment with ITF3756 reduced the mRNA level of Pd-1 and Lag-3. Gene expression of Tim-3 was also downmodulated, but this effect did not result in reduction of protein expression at the time of detection. ITF3756 reduced the expression of t-bet (Tbx21) driving $T$ effector differentiation and increased genes related to $\mathrm{T}$ memory phenotype (Eomes, Lef-1 and albeit slightly, Tcf-7). T cell activation requires a metabolic reprogramming that supports highly proliferative phenotype and $\mathrm{T}$ effector differentiation. ITF3756 treatment decreased both Hif- $1 \alpha$ and Glut-1 gene expression that are associated with TCR activation during the exhaustion process. $\mathrm{T}$ central memory cells produce less cytokines compared to $\mathrm{T}$ effector and effector memory cells. ITF3756 treatment decreased the genes expression of Il-2, Ifn$\gamma$ and Tnf- $\alpha$. All these effects resulted dose dependent.

Conclusions The selective inhibitor of HDAC6 ITF3756 delays the terminal differentiation of CD8 T cells and increases the percentage of memory $\mathrm{T}$ cells with a reduced expression of exhaustion markers in vitro. These results are the basis to further explore the possible use of ITF3756 as a safe ex vivo treatment of CD8 T cells for adoptive cell transfer.

Disclosure Information C. Ripamonti: A. Employment (full or part-time); Significant; Italfarmaco SpA. C. Steinkuhler: A. Employment (full or part-time); Significant; Italfarmaco SpA. G. Fossati: A. Employment (full or part-time); Significant; Italfarmaco SpA.

\section{P02 Microbiome and Immune System/ Immunotherapy}

\section{P02.01 PREDICTIVE IMPACT OF THE GUT MICROBIOTA ON TREATMENT RESPONSE TO CD19 SPECIFIC CAR T-CELLS}

\begin{abstract}
${ }^{1,2}$ V Blumenberg* ${ }^{3} \mathrm{E}$ Zamir, ${ }^{3} \mathrm{~S} S \mathrm{Schmidt},{ }^{3} \mathrm{R}$ Gaiser, ${ }^{3} \mathrm{~N}$ Cullin, ${ }^{1,2} \mathrm{~V}$ Bücklein, ${ }^{1,2} \mathrm{C}$ Schmidt, ${ }^{1} \mathrm{M}$ von Bergwelt, ${ }^{3} \mathrm{E}$ Elinav, ${ }^{3} \mathrm{CK}$ Stein-Thoeringer, ${ }^{1,2,4} \mathrm{M}$ Subklewe. ${ }^{1}$ University Hospital, LMU Munich, Munich, Germany; ${ }^{2}$ Laboratory for Translational Cancer Immunology, Gene Center of the LMU Munich, Munich, Germany; ${ }^{3}$ Research Division Microbiome and Cancer, German Cancer Research Center (DKFZ), Heidelberg, Germany; ${ }^{4}$ German Cancer Consortium (DKTK) and German Cancer Research Center (DKFZ), Heidelberg, Germany
\end{abstract}

\subsection{6/jitc-2020-ITOC7.37}

Background High response rates (RR) have led to the approval of the CD19 specific CAR T-cell products AxicabtageneCiloleucel and Tisagenlecleucel for the treatment of refractory/relapsed B-cell precursor ALL (BCP-ALL) and Diffuse Large B-cell lymphoma (DLBCL). However, only a subgroup of patients achieves long-term remission. Additionally, most patients experience adverse effects such as cytokine release syndrome or neurotoxicity. Therefore, we need to better understand mechanisms of relapse and disease progression or toxicity to improve effectiveness of CD19 CAR T-cell therapy. As the gut microbiota plays an important role in modulating T-cell based immunotherapy, we hypothesize, that its signature also impacts clinical outcomes of CAR Tcell therapy.

Materials and Methods We are currently collecting and 16S rRNA sequencing fecal biospecimen from BCP-ALL and DLBCL patients before, during and after treatment with Axicabtagene-Ciloleucel and Tisagenlecleucel at the University
Hospital of the LMU. Microbiota data are integrated into a patient-centered 'hospitalome' including onset and type of infection and of immunotoxicity, concomitant anti-infective and immunosuppressive agents as well as response to CAR Tcell therapy.

Results Preliminary data analysis revealed, that 4-14 days after CAR T-cell infusion the alpha diversity of the gut microbiome of each patient decreases drastically, whereas pre-lymphodepletion gut microbiota has a high diversity. Furthermore, the microbiota composition during the course of treatment changes as seen by beta diversity changes. In more detail, after CAR T-cell infusion, we observed instances of gut microbiota mono-domination with Enterococci or the genus Rikinella.

Conclusions The gut microbiome of patients being treated with CAR T cells undergoes large and diverse compositional changes. We currently explore how this microbiome heterogeneity relates to the distinct responses and immunotoxicity of patients after CAR T-cell therapy. Hence, this study will enable microbiome-based stratification of patients, in order to predict and improve patient outcome to this personalized Tcell recruiting immunotherapy approach.

Disclosure Information V. Blumenberg: None. E. Zamir: None. S. Schmidt: None. R. Gaiser: None. N. Cullin: None. V. Bücklein: None. C. Schmidt: None. M. von Bergwelt: None. E. Elinav: None. C.K. Stein-Thoeringer: None. M. Subklewe: None.

\section{P02.02 GENERATING NEO- AND SELF-ANTIGEN SCREENING LIBRARIES FOR CLASS II HLA PRESENTATION}

${ }^{1,2} \mathrm{~V}$ Pinamonti ${ }^{*},{ }^{3} \mathrm{~N}$ Felix, ${ }^{1} \mathrm{JM}$ Lindner. ${ }^{1}$ BioMed $X$, Heidelberg, Germany; ${ }^{2}$ University of Heidelberg, Heidelberg, Germany; ${ }^{3}$ Janssen, Spring House, PA, USA

\subsection{6/jitc-2020-ITOC7.38}

Background The identification of neo-antigens presented by tumor cells is an essential tool for cancer prevention, diagnosis, and therapy. Current approaches frequently involve mass spectrometric analysis, but these workflows do not concomitantly identify the cognate T-cell receptor. Likewise, TCR functional screens are often limited to a subset of predicted neo-epitopes.

Materials and Methods Here, we present a new method for the generation of an un-biased antigen-presenting library. Due to the genomic instability of tumors, patient-specific libraries will be cloned using random primers, ensuring the cloning of tumor-specific transcribed regions. This approach will not only address class I presentation of intracellular tumor antigens, but is also designed to simultaneously screen for cross-presentation on class II MHC complexes by professional antigen-presenting cells, an increasingly important component of anti-tumor immune responses. To guarantee presentation of genetically encoded antigens on class II MHC complexes, a signal motif for chaperone-mediated autophagy (CMA) is introduced in front of the cDNA sequence. Furthermore, antigens will be processed by the intracellular machinery, avoiding potential restrictions on spliced peptides.

Conclusions Once established, these libraries can be exploited in high-throughput screens to functionally identify neo-antigens together with their corresponding T-cell receptor.

Disclosure Information V. Pinamonti: Other; Significant; Janssen. N. Felix: Other; Significant; Janssen. J.M. Lindner: Other; Significant; Janssen. 